\title{
Bianchi Type IX Magnetized Bulk Viscous String Cosmological Model in General Relativity
}

\author{
V. G. Mete', V. D. Elkar², V.S. Deshmukh³ \\ ${ }^{1}$ Department of Mathematics, R.D.I.K. \& K.D. College, Badnera- Amravati (M.S.), India \\ ${ }^{2}$ Department of Mathematics, J.D.Patil Sangludkar Mahavidyalaya, Daryapur, Dist. Amravati, India \\ ${ }^{3}$ Department of Mathematics, P.R.M.I.T.\&R. , Badnera- Amravati (M.S.), India \\ Email: vmete5622@gmail.com
}

\begin{abstract}
In this paper we present a bulk viscous magnetized Bianchi type IX string cosmological model in general relativity. In order to find determinate solution of the investigated model, we assume the conditions :(i) Scalar expansion is proportional to shear scalar and (ii) $\xi \theta=$ constant, where $\xi$ is bulk viscosity and $\theta$ is scalar expansion. Some physical and geometrical properties of the investigated model are also discussed.
\end{abstract}

Keywords: Bianchi type IX space time, bulk viscous, magnetized cosmic string.

\section{Introduction}

It is well known that Einstein's general theory of relativity has been successful in finding different models for the universe. Einstein's theory of general relativity is considered as the only successful theory of gravitation in terms of geometry which has the most beautiful structure of the theoretical physics. In recent years, Bianchi type-IX cosmological models are very popular for relativistic studies. These models are also used to examine the role of certain anisotropic sources during the formation of large scale structure as we see the universe today. Many researchers have taken keen interest in studying Bianchi Type IX universes, because familiar solutions like Robertson Walker universe with positive curvature, the de-Sitter universe, the Taub-Nat solutions etc. are particular case of Bianchi Type IX space time. Number of authors Chakraborty [1], Raj Bali and Dave[2], Raj Bali and Yadav [3] have studied Bianchi type-IX string as well as viscous fluid models in general relativity. Pradhan [4] have studied some homogeneous Bianchi type-IX viscous fluid cosmological model with varying cosmological constant.

On the other hand, Bulk viscosity plays a significant role in the early evolution of the universe and contributes to the accelerated expansion phase of the universe popularly known as the inflationary phase. The effect of viscosity on the evolution of cosmological models and the role of viscosity in avoiding the initial big bang singularity have been studied by several authors [5-6]. In late nineties, two teams studying distant type Ia-supernovae (SNe-Ia) independently presented evidence of expansion [7-8] and confirmed later by cross checks from the cosmic microwave background radiation (CMBR)[9] and large scale structure (LSS) [10-13].

In addition, the magnetic field has important role at the cosmological scale and is present in galactic and intergalactic space. It plays a vital role in the description of the energy distribution in the universe as it contains highly ionized matter. Strong magnetic fields can be created due to adiabatic compression in cluster of galaxies. Large scale magnetic fields give rise to anisotropies in the universe. Primordial magnetic fields of cosmological origin have been discussed by Asseo et.al [14] and Madsen [15]. Melvin [16] suggested, in the cosmological solution for dust and electromagnetic fields, that during the evolution of the universe, the matter was in highly ionized state and smoothly coupled with electromagnetic and consequently formed a neutral matter as a result of expansion of the universe. Hence, in a string dust universe the presence of magnetic fields is not unrealistic. The magnetic field has the significant role in the dynamics of the universe depending on the direction of the field lines [17-18]. Tyagi et.al.[19] has studied homogeneous anisotropic Bianchi type-IX cosmological models for perfect fluid distribution with electromagnetic field. Recently Tyagi and Singh.[20], Ghate and Sontakke [21-22] have investigated Bianchi type-IX cosmological models in different context. Mete and Elkar [23] have studied Bianchi 
type-IX cosmological models for viscous fluid with electromagnetic field and time dependent $\Lambda$-term. Recently Bianchi type- $\mathrm{V}$ magnetized cosmological model with wet dark fluid in general relativity has been studied by Mete et.al [24].

Motivated by the above discussions, in this paper, we have focused upon the problem of establishing a formalism for studying a new integrability of magnetized Bianchi type IX bulk viscous string cosmological models in general relativity. The behaviors of the models in the presence magnetic fields are also discussed.

\section{$2 \quad$ Metric and Field Equations}

We consider Bianchi type IX space time in the form

$$
d s^{2}=-d t^{2}+A^{2} d x^{2}+B^{2} d y^{2}+\left(B^{2} \sin ^{2} y+A^{2} \cos ^{2} y\right) d z^{2}-2 A^{2} \cos y d x d z
$$

where $A$ and $B$ are scale factors and are functions of cosmic time $t$.

The energy momentum tensor for a cloud string with a magnetic field in a co-moving coordinate system is

$$
T_{i j}=\rho u_{i} u_{j}-\lambda x_{i} x_{j}-\xi \theta\left(u_{i} u_{j}+g_{i j}\right)+E_{i j}
$$

where the vector $u_{i}$ describes the cloud 4 -velocity and $x_{i}$ represents a direction of anisotropy, i.e. the string satisfies the relations

$$
u^{i} u_{i}=-x^{i} x_{i}=-1, u^{i} x_{i}=0
$$

Here $\rho$ is the rest energy of the cloud strings with massive particles attached to them. It is given by $\rho=\rho_{p}+\lambda$, where $\rho_{p}$ being the rest energy density of particles attached to the strings and $\lambda$ is the density of tension that characterizes the strings. The energy momentum for the magnetic field is

$$
E_{i}^{j}=\frac{1}{4 \pi}\left(-F_{i l} F_{j m} g^{l m}+\frac{1}{4} g_{i}^{j} F_{l m} F^{l m}\right)
$$

where $E_{i}^{j}$ is the electromagnetic field tensor satisfying Maxwell's equations

$$
F_{i j ; k}+F_{j k ; l}+F_{k i ; j}=0 \text { or } \frac{\partial}{\partial x^{j}}\left(F^{i j} \sqrt{-g}\right)=0
$$

In comoving coordinates, the incident magnetic field is taken along x-axis. With the help of Maxwell's equations (5), the only nonvanishing component of $F_{i j}$ is

$$
F_{23}=H \sin y=\text { constant }
$$

In order to determine the system completely, we consider Takabayasi's equation of state [25],

$$
\rho=l \lambda
$$

where $l$ is constant.

The Einstein's field equations (in gravitational unit $c=1, G=1$ ) read as

$$
R_{i j}-\frac{1}{2} g_{i j} R=-8 \pi T_{i j}
$$

For the metric(1), the above field equations with the help of equations (2)- (7) takes the form

$$
\begin{gathered}
\frac{2 B_{44}}{B}+\frac{B_{4}^{2}}{B^{2}}+\frac{1}{B^{2}}-\frac{3}{4} \frac{A^{2}}{B^{4}}=\lambda+\xi \theta-\frac{H^{2}}{8 \pi B^{4}} \\
\frac{A_{44}}{A}+\frac{B_{44}}{B}+\frac{A_{4} B_{4}}{A B}+\frac{1}{4} \frac{A^{2}}{B^{4}}=\xi \theta+\frac{H^{2}}{8 \pi B^{4}} \\
\frac{2 A_{4} B_{4}}{A B}+\frac{B_{4}^{2}}{B^{2}}+\frac{1}{B^{2}}-\frac{1}{4} \frac{A^{2}}{B^{4}}=\rho-\frac{H^{2}}{8 \pi B^{4}}
\end{gathered}
$$

\section{$3 \quad$ Solution in Presence of Bulk Viscosity and Magnetic Field}

The field equations (9) to (11) are a system of three highly non-linear differential equations with five 
unknown parameters $A, B, \xi, \lambda, \rho$. The system is thus initially undetermined. To obtain a deterministic solution, the following physical conditions are used.

Firstly, we assume that the coefficient of bulk viscosity $\xi$ is inversely proportional to the expansion. This condition leads to

$$
\xi \theta=k \text { (constant) }
$$

The motive behind assuming this condition is explained in the literature [26-28]. Secondly, we consider the expansion scalar $\theta$ is proportional to the shear $\sigma$, i.e. $\theta \propto \sigma[29]$.

This condition leads to

$$
A=B^{n}
$$

where $n \succ 0$ a constant.

From equations (9)-(11), with the help of (12) and (13), eliminating $\lambda$, we have

$$
B_{44}+\alpha \frac{B_{4}^{2}}{B}=\frac{\beta}{B}+\gamma B^{2 n-3}+\frac{\delta}{B^{3}}+\frac{k}{2} B
$$

where

$$
\alpha=\frac{l-(2 n+1)}{2 l}, \beta=\frac{1-l}{2 l}, \gamma=\left(\frac{3 l}{4}-\frac{1}{4}\right) \frac{1}{2 l}, \delta=\frac{(1-l) L}{2 l} .
$$

To solve equation (14), we denote $B_{4}=f(B)$ then $B_{44}=\frac{d f(B)}{d B} f(B)$ and equation (14) can be reduced to the linear differential equation in the form

$$
\frac{d}{d B} f^{2}(B)+2 \alpha \frac{f^{2}(B)}{B}=Q
$$

where $Q=\frac{\beta}{B}+\gamma B^{2 n-3}+\frac{\delta}{B^{3}}+\frac{k}{2} B$

Integrating equation (15), we obtain

Thus we get,

$$
f^{2}(B)=\frac{\beta}{2 \alpha}+\gamma \frac{B^{2(n-1)}}{2 n+2 \alpha-2}+\delta \frac{B^{-2}}{2 \alpha-2}+\frac{k}{2} \frac{B^{2}}{2 \alpha+2}+\frac{C}{B^{2 \alpha}}
$$

$$
d t=\left[\frac{\beta}{2 \alpha}+\gamma \frac{B^{2(n-1)}}{2 n+2 \alpha-2}+\delta \frac{B^{-2}}{2 \alpha-2}+\frac{k}{2} \frac{B^{2}}{2 \alpha+2}+\frac{C}{B^{2 \alpha}}\right]^{\frac{-1}{2}} d B
$$

where $C$ is constant of integration.

Hence line element (1) becomes

$$
\begin{aligned}
& d s^{2}=\left[\frac{\beta}{2 \alpha}+\frac{\gamma B^{2(n-1)}}{2 n+2 \alpha-2}+\frac{\delta B^{-2}}{2 \alpha-2}+\frac{k}{2} \frac{B^{2}}{2 \alpha+2}+\frac{C}{B^{2 \alpha}}\right]^{-1} d B^{2} \\
& +B^{2 n} d x^{2}+B^{2} d y^{2}+\left(B^{2} \sin ^{2} y+B^{2 n} \cos ^{2} y\right) d z^{2}-2 B^{2 n} \cos y d x d z
\end{aligned}
$$

Using the suitable transformation of coordinates equation (18) reduces to

$$
\begin{aligned}
& d s^{2}=\left[\frac{\beta}{2 \alpha}+\frac{\gamma T^{2(n-1)}}{2 n+2 \alpha-2}+\frac{\delta T^{-2}}{2 \alpha-2}+\frac{k}{2} \frac{T^{2}}{2 \alpha+2}+\frac{C}{T^{2 \alpha}}\right]^{-1} d T^{2} \\
& +T^{2 n} d x^{2}+T^{2} d y^{2}+\left(T^{2} \sin ^{2} y+T^{2 n} \cos ^{2} y\right) d z^{2}-2 T^{2 n} \cos y d x d z
\end{aligned}
$$

where $B=T$ can be determined by equation (19).

\section{Some Physical Properties of the Model}

For the model (19), the physical parameters: the energy density $(\rho)$, the string tension density $(\lambda)$, the particle energy density $\left(\rho_{p}\right)$, the coefficient of bulk viscosity $(\xi)$, the spatial volume $(V)$, expansion scalar $(\theta)$ and shear scalar $\left(\sigma^{2}\right)$, the Hubble parameter $(H)$ and the deceleration parameter $(q)$ are 
obtained as follows:

$$
\rho=\left\{\left[\frac{(2 n+1) \beta}{2 \alpha}+1\right]+\left[\frac{\gamma(2 n+1)}{2 n+2 \alpha-2}-\frac{1}{4}\right] T^{2(n-1)}+\left[\frac{(2 n+1) \delta}{2 \alpha-2}+L\right] T^{-2}+C(2 n+1) T^{-2 \alpha}\right\} T^{-2}+M(20)
$$

where $L=\frac{H^{2}}{8 \pi}, \quad M=\frac{k(2 n+1)}{2(2 \alpha+2)}$

$$
\begin{gathered}
\lambda=\frac{1}{l} \rho \\
\rho_{p}=\rho-\lambda=\left(1-\frac{1}{l}\right) \rho \\
\xi=\frac{k}{\theta}=\frac{k}{n+2}\left(\frac{\beta}{2 \alpha} T^{-2}+\frac{\gamma}{2 n+2 \alpha-2} T^{2(n-2)}+\frac{\delta}{2 \alpha-2} T^{-4}+C T^{-2 \alpha-2}+\frac{k}{2(2 \alpha+2)}\right)^{\frac{-1}{2}} \\
\theta=\frac{A_{4}}{A}+2 \frac{B_{4}}{B}=(n+2)\left(\frac{\beta}{2 \alpha} T^{-2}+\frac{\gamma}{2 n+2} \sin y\right. \\
\sigma^{2}=\frac{2}{3}\left(\frac{A_{4}}{A}-\frac{B_{4}}{B}\right)^{2}=\frac{2}{3}(n-1)^{2}\left(\frac{\beta}{2 \alpha} T^{-2}+\frac{\gamma}{2 n+2 \alpha-2}+\frac{\delta}{2 \alpha-2} T^{-4}+C T^{-2 \alpha-2}+\frac{k}{2(2 \alpha+2)}\right)^{\frac{1}{2}} \\
\left.T^{2(n-2)}+\frac{\delta}{2 \alpha-2} T^{-4}+C T^{-2 \alpha-2}+\frac{k}{2(2 \alpha+2)}\right)
\end{gathered}
$$

Hence

$$
\lim _{T \rightarrow \infty}\left(\frac{\sigma^{2}}{\theta^{2}}\right)=\frac{2(n-1)^{2}}{3(n+2)^{2}}=\text { constant }
$$

Here we observe that when $T \rightarrow 0, \quad \sigma \rightarrow \infty$ and $\quad \sigma / \theta=$ constant, as $T \rightarrow \infty$.

The Hubble parameter:

$$
H=\frac{\theta}{3}=\frac{(n+2)}{3}\left(\frac{\beta}{2 \alpha} T^{-2}+\frac{\gamma}{2 n+2 \alpha-2} T^{2(n-2)}+\frac{\delta}{2 \alpha-2} T^{-4}+C T^{-2 \alpha-2}+\frac{k}{2(2 \alpha+2)}\right)^{\frac{1}{2}}
$$

and the deceleration parameter $(q)$

$$
q=-1+\frac{3}{(n+2)} \frac{\left[\frac{\beta}{2 \alpha} T^{-3}+\frac{\gamma(n-2)}{2 n+2 \alpha-2} T^{2 n-5}-\frac{2 \delta}{2 \alpha-2}+C(-\alpha-1) T^{-2 \alpha-3}\right]}{\left[\frac{\beta}{2 \alpha} T^{-2}+\frac{\gamma}{2 n+2 \alpha-2} T^{2(n-2)}+\frac{\delta}{2 \alpha-2} T^{-4}+\frac{k}{2(2 \alpha+2)}+C T^{-2 \alpha-2}\right]^{3 / 2}}
$$

\section{Conclusion}

In this paper, we have studied magnetized Bianchi type-IX bulk viscous string cosmological model in general relativity. To get the deterministic model we have assumed two conditions. Firstly coefficient of bulk viscosity is inversely proportional to expansion scalar. i.e. $\xi \theta=$ constant and secondly expansion scalar is proportional to the shear scalar, i.e. $\theta \propto \sigma$. We observe that the spatial volume $V \rightarrow 0$ when $T \rightarrow 0$ and $V \rightarrow \infty$ when $T \rightarrow \infty$, thus the model is expanding. For the anisotropy when $T=\infty$, $\sigma / \theta \neq 0$, this implies that the model does not approach isotropy at late time when $-2<n<2$. Also, for $n=1$ the shear scalar $\sigma$ is zero and $\frac{\sigma^{2}}{\theta^{2}} \rightarrow 0$ as $T \rightarrow \infty$. Thus the model becomes isotropic at late time when $-2<n<2$. The shear scalar does not tend to zero faster than expansion at late time. 
Acknowledgement. The author thanks an unknown reviewer for helpful advices which permitted to improve this work.

\section{References}

1. S. Chakraborty , "A study on Bianchi IX cosmological model", Astrophysics and Space Science,180 (2), 293303,1991

2. Raj Bali and S. Dave, "Bianchi Type-IX string cosmological model in general relativity", Pramana J. Phy., 56 (4), $513-518,2001$

3. Raj Bali and M.K.Yadav, "Bianchi Type-IX viscous fluid cosmological model in general Relativity", Pramana J. Phy., 64 (2), 187-196, 2005

4. A.Pradhan, S.K. Srivastav and M.K.Yadav, "Some homogeneous Bianchi type- IX viscous fluid cosmological models with a varying $\Lambda "$, Astrophysics and Space Science;298,419- 432,2005

5. Shri Ram and C.P.Singh,, "Anisotropic Bianchi Type -II Cosmological Models in Self Creation Cosmology", Astrophy. Spa. Sci.,257,287, 1998

6. A. Pradhan and H.R. Pandey,"Bulk Viscous Cosmological Models in Barber's Second Self Creation Theory", Int. J. Mod. Phy. , 2002

7. A.G. Riess et al., "Type Ia Supernova Discoveries at z ?1 from the Hubble Space Telescope: Evidence for the Past Deceleration and Constraints on Dark Energy Evolution", The Astrophys. J., 607(2), 665-678, 2004

8. D.N. Spergel et al., "Type Ia Supernova Discoveries at z ?1 from the Hubble Space Telescope: Evidence for the Past Deceleration and Constraints on Dark Energy Evolution", Astrophys. J. Suppl. Ser., 170(2), 377- 385,2007

9. D.N. Spergel et al., "First-YearWilkinson Microwave Anisotropy Probe (WMAP)Observations: Determinations of cosmological parameters", Astrophys. J. Suppl. Ser., 148(1),175-182, 2003

10. E.Hawkins et al., "The $2 \mathrm{dF}$ galaxy redshift survey: correlation functions, peculiar velocities and the matter density of the universe", Mon. Notices of the Royal Astrono.Soc., 346(1), 78-96,2003

11. K. Abazajian et. al., "The First Data Release of the Sloan Digital Sky Survey", Astronom. J., 128, 502-511, 2004

12. K. Abazajian et .al., "The Second Data Release of the Sloan Digital Sky Survey", Phy. Rev. D, 69, 103112,2004

13. M.Tegmark et.al.,"Cosmological parameters from SDSS and WMAP", Phys. Rev. D, 69(10),103-501, 2004

14. E. Asseoet.al. "Extra galactic magnetic fields". Phys. Rep. Sol, H. Phys. Rep. 148,307-436, 1987

15. M.S.Madsen, "Magnetic field in cosmology", Not. R. Astron. 237, 109-117, 1989

16. M. A. Melvin, "Homogeneous axial cosmologies with electromagnetic field and dust",Ann. New York Acad. Sci. 262, 253-274, 1975

17. K.L. Mahanta and A.K. Biswal, Rom. Journ. Phys., 58 (3-4) , 239-246,2013

18. E.J. King and P Coles, "Dynamics of a magnetized Bianchi-I Universe with Vacuum Energy", Classical and Quantum Gravity, 24, 2061-2072,2007

19. A .Tyagi and D. Chhajed, "Homogeneous anisotropic Bianchi type -IX cosmological model for perfect fluid distribution with electromagnetic field", American J. Math. and Statis.", 2 (3),19-21, 2012

20. A. Tyagi, G.P. Singh, "Magnetized bulk viscous Bianchi type -IX cosmological model with varying $\Lambda$ " , Ultra Scientist ,22(2), 658-664,2010.

21. H.R. Ghate and A.S. Sontakke, "Bianchi type IX cosmological models with a anisotropic dark energy", International Journal of Scientific and Engineering Research, 4(6), 769-774, 2013

22. H.R. Ghate and A.S. Sontakke, "Binary mixture of Anisotropic dark energy and Perfectfluid in Bianchi TypeIX space-time", JPMS, 3(2) ,122-131,2013.

23. V.G.Mete and V.D.Elkar, "Bianchi type-IX cosmological model for viscous fluid with electromagnetic field and time dependent $\Lambda$ term", Prespacetime Journal 6 (11), pp-1157-1164, 2015.

24. V.G.Mete, K.R.Mule and V.D.Elkar, "Bianchi type-V magnetized cosmological model with wet dark fluid in general relativity" , Int. J. of current research , 8(11) , 41464-41468, 2016

25. T. Takabayasi "Quantum Mechanics, Determination, Causality and Particles", Reidal: Dordrecht, the Netherlands, 179, 1976

26. RajBali and A. Pradhan, "Bianchi type III string cosmological models with time dependent bulk viscosity", Chin. Phys. Lett.24, 585-588, 2007 
27. B. Saha, "Bianchi type-I universe with viscous fluid", Modern physics letters A, 20:28 , 2127-2143,2012

28. V. K Yadav and L. Yadav, "Some Bulk Viscous Magnetized LRS Bianchi Type-I StringCosmological Models in Lyra's Geometry", Rom. J. Phys. 58,64-74,2013

29. R. K. Tiwari and Dwivedi, "Kantowski-Sachs cosmological models with time-varying $g$ and $\lambda$, $u$ ", fizika (zegreb),19, 1-8, 2010. 